CUPAUAM. 19-1992, 245-264

\title{
EL ACUEDUCTO ROMANO DE UCUBI (ESPEJO, CORDOBA)
}

\author{
LOURDES ROLDAN GOMEZ \\ UNIVERSIDAD AUTONOMA DE MADRID
}

\begin{abstract}
Resumen
El presente trabajo se basa en el seguimiento, documentación y estudio constructivo de un acueducto recientemente valorado en la arqueología cordobesa, el que, desde las fuentes cercanas a Nueva Carteya, llevaba las aguas hasta la ciudad de Ucubi (Espejo). Con ello, aportamos un nuevo documento para el estudio de las técnicas constructivas romanas en la Península, valorando la utilización de materiales constructivos característicamente romanos.
\end{abstract}

\section{Summary}

The present work is based on the documentation and constructive study, of an aqueduc recently valuated in the archaeology of Cordoba, that from the sources near to New Carteya, where taken to the city of Ucubi (Espejo). With this we have tried to bring a new document for the study of roman constructive technics in the Peninsula, valuing the utilisation of the caracteristic roman materials.

El acueducto de Nueva Carteya discurre por tierras cordobesas en los términos Municipales de Nueva Carteya, Castro del Río y Espejo. Su misión era, abastecer de agua a la antigua ciudad de Espejo, colonia Claritas Iulia Ucubi.

El conocimiento de este acueducto parte del siglo pasado(1), pero su estudio es muy posterior. Aparecen citados algunos de sus tramos en el Catálogo Artístico y Monumental de la provincia de Córdoba en 1985 (2) y, más recientemente, se ha ocupado del mismo P. Lacort Navarro (Lacort y Jiménez, 1988; Lacort, 1988, 1989 y 1991).

(1) La noticia de su descubrimiento en 1807 fue dada por L. M. Ramírez de las Casas Deza en su Chorografia de 1842 (Marcos Pous y Vicent Zaragoza, 1989, 193; Lacort Navarro, 1991, 175). No está recogida esta referencia a Espejo en la publicación de 1986 (Ramírez de las Casas Deza, 1986).

(2) Concretamente en el tramo correspondiente al T. M. de Castro del Rio, cfr. Catálogo Artístico y Monumental de la provincia de Córdoba T.II, Córdoba 1985, 39. 
Su recorrido a lo largo de una distancia de unos $15 \mathrm{Km}$. en línea recta, transportaba el agua desde fuentes cercanas a la ciudad de Nueva Carteya hasta la citada Ucubi yendo, como es habitual en los acueductos romanos, en busca de las aguas más convenientes aunque estuvieran alejadas de la ciudad (Leveau, 1979).

El acueducto de Nueva Carteya tenía un trazado en dirección norte o noroeste hacia Espejo, de modo que, por la envergadura de la obra debió realizarse para abastecer a Ucubi y llegaría a la ciudad antigua de Espejo desde el sur(3).

La construcción del acueducto es sencilla evitando, en lo que actualmente se documenta, la realización de arquationes u obras de envergadura mediante la solución de rodear las vaguadas, siguiendo las curvas de nivel. De este modo, la pendiente del recorrido en su parte central (la mejor documentada por el momento) se mantenía suave y continua (prácticamente constante) exceptuando la zona de recogida de aguas, a mayor altura.

A su llegada a la ciudad, la conducción tendría que remontar el desnivel existente entre ésta y el terreno circundante aunque la ausencia de restos documentados en esta zona ha impedido la confirmación del tramo final.

A pesar de la sencillez del recorrido se emplearon soluciones técnicas adecuadas, como se documenta en el sistema de captación de aguas, o en el empleo de arcos individuales en los casos en que se requería atravesar pequeñas vaguadas. De este modo, se consiguió el recorrido más lógico y, probablemente, el más adecuado a las posibilidades económicas de la ciudad.

La conducción discurre, en su mayor parte, sobre un pequeño murete, no muy elevado sobre el suelo, realizado sistemáticamente en opus caementicium sin paramento externo. En ocasiones, el acueducto se introduce bajo la tierra probablemente manteniendo el mismo tipo de obra.

La medida del specus es prácticamente constante en el recorrido conocido, excepto en el caso de que se trate de canales de aportación en los que disminuye sensiblemente la sección. En ambos casos existe una protección interior de opus signinum aunque sólo en el canal principal se documentan los característicos modillones hidráulicos.

La descripción del acueducto que a continuación exponemos corresponde a la primera parte y tramo central de su recorrido: desde los canales de aportación, en la cabecera del mismo, hasta que alcanza la zona más baja que rodea la ciudad de Espejo. A partir de aquí no hemos encontrado vestigios, por lo que podría pensarse que, debido al carácter más llano del terreno y a las continuas labores agrícolas, hayan sido destruidos (4).

(3) Aunque su entrada a la ciudad se hizo, al parecer, por el noreste ya que, en este lado, se cita la presencia de un gran depósito mencionado por Ramírez de Arellano en 1904 (Ramírez de Arellano, 1983, 374) y que P. Lacort considera un lacus (Lacort, 1988, 177).

(4) El tramo documentado es claramente visible en varios de sus fragmentos, entre los olivares de la zona. En ocasiones ha sido destruido por las labores agrícolas y en otros tramos muestra claras señales de haber sido intencionadamente retirada la tierra que lo cubria e incluso limpiado el interior del specus, al parecer estas operaciones han sido realizadas por mera curiosidad y sin que por el momento se hayan producido graves dańos de forma intencionada. No obstante, la situación del acueducto es propicia para que esto pueda suceder en el futuro. 


\section{I.- EL RECORRIDO}

\section{1.- Los canales de aportación}

El Acueducto de Nueva Carteya recogía en su tramo inicial aportes de agua de diversos canales, de dimensiones más pequeñas que el canal principal (Fig.l). Estos canales se nutrían probablemente en las fuentes de los pequeños arroyos que van a desembocar al Carchena por su margen izquierda y se juntarían con el canal principal en la zona baja, muy cerca de Nueva Carteya (5). Es común en los acueductos romanos la existencia de varios canales de aporte que generalmente desembocaban en un depósito colector cubierto y revestido de hormigón hidráulico, como ocurre en Arles y en Lyon (Leveau, 1979, 10).

Los restos constructivos aparecen en varios lugares, y se documentan en tres puntos fragmentos del specus (Fig. 1).

Cerca de las fuentes que corresponden al Arroyo de la Mora (junto al Cortijo Ortega), a unos 2 Kilómetros al Sureste de Nueva Carteya, se documenta un fragmento de specus in situ de dimensiones más pequeñas que el canal principal (Pto. 22) (Fig. 5 a y b) y más arriba, a unos $580 \mathrm{~m}$. de altura, una fuente o aljibe que aún se utiliza en la actualidad.

Hacia la zona baja, junto a la carretera de Nueva Carteya a Dña. Mencía y muy cerca del mismo arroyo, existen nuevos restos constructivos de difícil asignación. Se trata de un frogón de hormigón, in situ, que no parece ser resto de canalización (sus dimensiones son mucho mayores que los pequeños canales) aunque debe de tratarse de alguna construcción relacionada con el acueducto que quizás correspondería a un punto de unión de los canales de aportación.

En las cercanías del Arroyo Molinillos existen también restos de otro canal de formación (Fig. 1) que recogería, probablemente, el agua de la Fuente de las Espejas en la cabecera del Arroyo y que se documenta en varios puntos.

El primero de ellos se trata de un fragmento de specus (de $1,56 \mathrm{~m}$. de largo total y $0,89 \mathrm{~m}$. de anchura total) (Pto. 23) (Fig. 6) y se encuentra a media ladera, en una zona de olivos (6). Ya más cerca de Nueva Carteya, en el cruce del Arroyo Molinillos con un camino secundario, se documenta un nuevo fragmento del specus, el mejor conservado (Pto. 24). En este tramo el acueducto iría subterráneo y se ha podido observar en él tanto la forma de construcción del specus, similar a las anteriores, como la cubrición a base de tegulae en $\mathrm{V}$ y protegido con opus caementicium (Fig. $5 \mathrm{cyd}$ ). En las inmediaciones existe una fuente o aljibe aún hoy en uso.

Por último, conocemos la existencia de otros fragmentos, aún más arriba, cerca de la Fuente de las Espejas, junto a la carretera de Cabra. Hace pocos años se podían ver en este lugar (justo en el punto en que la carretera cruza un pequeño arroyo del Molinillos) fragmentos de hormigón de la cimentación y del specus que hoy han desaparecido (7).

(5) Una de estas fuentes de recogida, quizás la principal, podría ser la de Plaza de Armas que se sitúa al sur de Nueva Carteya, a $750 \mathrm{~m}$. de altura (Lacort y Jiménez, 1988, 14).

(6) El specus se realizó con alzados laterales independientes sobre una cimentación común, las paredes interiores se recubrieron de opus signinum que apoyaba sobre la parte superior de los muros y el fondo del specus se configuró mediante sucesivas capas de argamasa sin modillón hidráulico.

(7) Agradecemos a J. Serrano esta información así como su amabilidad al mostrarnos estos lugares y acompañarnos en el recorrido que realizamos para documentar los datos constructivos del acueducto. 


\section{2.- El Canal Principal}

El canal principal del acueducto se ha podido documentar con continuidad en un tramo de unos 3,5 Kilómetros desde Nueva Carteya hacia Espejo, atravesando el Término Municipal de Castro del Río (Fig. 2) (8).

El primer punto está a 1,5 Kilómetros al noroeste de Nueva Carteya. La conducción debía atravesar el pueblo, de forma subterránea, en dirección sureste-noroeste, ya que los canales de aporte están justamente al otro lado del mismo (9). El acueducto en el tramo inicial iría subterráneo. El modo de construcción sería probablemente semejante al de los canales de aportación y al resto del acueducto que, como veremos, es constante en el tramo que hemos podido documentar.

La conducción aparece en superficie en el punto medio de dos vaguadas que forman afluentes del Carchena por la margen derecha (Pto. 1). A partir de aquí se puede seguir su recorrido por tierras del Cortijo de Juan Frías, ceñido a la curva de los $440 \mathrm{~m}$. Se documenta un gran tramo a unos $300 \mathrm{~m}$. al noroeste del punto anterior (Pto. 2), tras cruzar el segundo de los citados arroyos y siguiendo la linde de dos bancales, que coinciden con la curva de nivel. De este modo, la cara este de la parte alta del acueducto enrasa con el nivel del terreno, lo que impide su estudio. Por el contrario, la cara oeste corresponde a otro bancal cuyo terreno baja cerca de $3 \mathrm{~m}$., facilitando así la descripción y el análisis de la construcción.

Dentro del tramo hay dos zonas que permiten estudiar la técnica constructiva; en la primera de ellas se ha vencido todo el lateral del specus dejando el interior del mismo y su cimentación al descubierto. En la segunda se conserva todavía el alzado exterior oeste con los revocos originales (lám. 1b).

A escasa distancia y en la misma cota del punto anterior se observan nuevos restos del acueducto que corresponden a un tramo de gran longitud (Pto. 3). Aunque no se documenta el recorrido en su totalidad, los lugares puntuales y tramos en los que aparece esporádicamente, no dejan lugar a dudas en cuanto a su recorrido. Se adapta perfectamente a la orografía del terreno, muy abrupto en esta zona, de modo que la conducción, que se dirigía hacia el noroeste, tiene que hacer dos giros de 180 grados (Pto. 3 al Pto. 9) para seguir la curva de nivel, tomando por fin la dirección norte. En el primero de ellos (Pto. 3) y en un punto en que atravesaba una vaguada se documenta, dentro de un tramo conservado de $34 \mathrm{~m}$., la existencia de un arco de ladrillos. De él se conservan únicamente los arranques de la rosca y parte del esqueleto del arco en opus caemenicium (Pto. 3.B) (lám. 2 a).

Más adelante, aún en las inmediaciones del Cortijo de Juan Frías, continua en dirección norte, siempre siguiendo la curva de $440 \mathrm{~m}$. El acueducto discurre ahora por tierra más llana, de olivares, y se puede ver ya el punto de destino, la actual Espejo, hacia donde se dirige prácticamente en línea recta y salvando las curvas de nivel (Pto.10 en adelante) (lám. 1a).

Esta parte (Pto. 10 al Pto. 13) es una de las más interesantes de la conducción. Aparece en varios puntos intermitentes y atravesando dos vaguadas. Se documenta un recorrido a lo largo de $300 \mathrm{~m}$. con una pronunciada curva aproximadamente en el centro del mismo. Antes, en el

(8) Es conocido el caput aquae cerca de la ciudad ibero-romana de la Plaza de Armas a $750 \mathrm{~m}$. de altura y el recorrido subterráneo de la conducción hasta la Fuente de la Mora y en el área de las Neverías hasta Nueva Carteya (Lacort, 1991, 176).

(9) Existian restos de la conducción subterránea (Lacort Navarro, 1991, 176), antes de llegar al pueblo junto al Arroyo Carchena y, también, en la Calle de San Pedro en Nueva Carteya se documentaron restos en el curso de algunas obras efectuadas hace tiempo. 
paso de la segunda vaguada, existe un gran tramo de unos $30 \mathrm{~m}$. que describe tres inflexiones en relación con el paso de la misma. La tercera de las inflexiones se alinea con la curva de circunvalación de la próxima loma, manteniendo así la cota.

El abancalamiento de la pendiente ha provocado que todo el lado este de la conducción en este punto aparezca totalmente saturado de tierra (lám. 2b). Por el contrario, se documenta mejor el lado oeste del specus, favorecido por la pendiente y, especialmente, en el tramo final de la vaguada. Todo este lado aparece vencido por la presión de la tierra, de forma que se observan desplazamientos de los bloques hasta 1,40 m., lo cual es lógico dado el tipo de construcción.

Al final de este tramo, justo antes del cambio de dirección por la curva aludida, se documenta la existencia de una pequeña arqueta de decantación perfectamente conservada y la única documentada por el momento en todo el recorrido del acueducto (lám. 3a).

Siguiendo esta alineación hacia el norte, entra en el Término Municipal de Castro del Río. En este punto se puede ver, cerca del linde entre ambos términos, dos tramos del acueducto que atraviesan sendas pequeñas vaguadas (Pto. 14 y Pto. 15); el segundo de ellos coincide con una zanja artificial que corre en dirección norte-sur junto al acueducto. De este modo, ha quedado al descubierto parte del mismo, por lo que se puede documentar el alzado de uno de los laterales, en una longitud de $29,5 \mathrm{~m}$. y con una altura de $0,82 \mathrm{~m}$. Una segunda zanja, perpendicular a la anterior, corta el acueducto transversalmente y permite documentar la sección completa: los dos muros laterales del specus con sus respectivos revocos.

A partir de aquí, escasos restos en las tierras del Cortijo del Piloncillo y del Calderón nos permiten, únicamente, constatar su recorrido hacia el norte en cota algo más baja que los tramos anteriores (curva de $420 \mathrm{~m}$.) (Pto. 16).

Desde el cruce de un curso de agua, aproximadamente $400 \mathrm{~m}$. al Norte del Cortijo del Calderón, se recupera de nuevo la línea del acueducto. Hemos podido ver la existencia de varios tramos (de no más de $3 \mathrm{~m}$.) (Pto. 17) en una zona muy alterada por el tractor que mantienen igual dirección e idéntica cota que los anteriores. Se trata de dos fragmentos de los laterales y de la base del specus. A continuación, el acueducto forma una amplia curva y atravesando un pequeño barranco cambia de dirección mediante un quiebro (Pto. 18 y Pto. 19). En estos puntos, aparte de pequeños fragmentos de la cimentación, se documenta un gran tramo, a ambos lados del quiebro del barranco, de 35 y $34 \mathrm{~m}$. respectivamente y con alzado de $0,96 \mathrm{~m}$. de altura media. Al final del mismo, el terreno actual enrasa en cota con la parte superior del acueducto haciéndolo desaparecer bajo tierra.

Por fin, tras un intervalo de unos $200 \mathrm{~m}$., cerca ya del Cortijo del Tomillar el acueducto sale de la zona más abrupta y va hacia el llano que rodea la actual Espejo. El tramo documentado presenta una longitud de unos $120 \mathrm{~m}$. (Pto. 20) y actualmente coincide con el linde de dos bancales aterrazados. De este modo, sólo se documenta la cara oeste de la cimentación, habiendo desaparecido todo el alzado del specus.

La distancia entre este punto y Espejo es de aproximadamente 6 Kilómetros en línea recta, a partir de aquí no hemos observado ningún resto más. El acueducto discurriría por tierras más bajas y llanas para remontar, llegando a Espejo, la altura de casi $400 \mathrm{~m}$.de la colina donde se ubica la ciudad. Desde allí, probablemente a partir de un castellum aquae (10), se distribuiría el agua a toda la ciudad.

(10) Como hemos dicho, Lacort interpreta como un depósito terminal los restos de un gran depósito circular, con muros de más de $1 \mathrm{~m}$. de grosor, construido a las afueras de la ciudad de Ucubi, hacia el noreste (Lacort, 1991, 177). 


\section{II.- CONSTRUCCION DEL ACUEDUCTO}

La construcción del Acueducto de Nueva Carteya se puede documentar, con bastante exactitud, a través de lo conservado del mismo. Aunque en algunos puntos solamente afloran sobre el terreno restos del opus caementicium de su cimentación existen otros en los cuales el alzado se conserva hasta una altura considerable (1,5 m.) (lám. 2c). En ellos hemos podido obtener datos suficientes para hacer una reconstrucción de su alzado y modo de realización.

La conducción estaba realizada en opus caementicium, tanto la cimentación como el alzado del specus, de piedras regulares de tamaño medio (entre 0,09 x 0,05 m. y 0,11 x $0,18 \mathrm{~m}$. con $0,09-0,12 \mathrm{~m}$. de profundidad) y trabadas con abundante argamasa. Esta técnica, que caracteriza la arquitectura romana, se utilizó en Pompeya y otras ciudades itálicas como Alba Fucens o Cosa, desde el s.III a.C. y en Roma a finales de este mismo siglo (Lugli 1957, 278 y 383; Giuliani, 1990, 166). Con posterioridad su uso fue habitual en cimentaciones, para núcleos de muros con paramentos en otros materiales, y en la construcción de bóvedas. Su empleo en alzado sin ningún tipo de paramento externo, tal y como lo encontramos en el acueducto de Nueva Carteya fue menos común. En la Bética, como en otras provincias del Imperio, la utilización de esta técnica fue más tardía. Se documenta en el Lacio a finales del s.II a.C y s.I a.C., donde se utilizó en los santuarios helenísticos de Praeneste, Tivoli y Terracina (Gullini, 1989, 70 ss.Coarelli, 19). El aprovechamiento de esta técnica en otras construcciones de La Galia y Britania o en Asia Menor no fue tan intenso como en Roma. Se puede apreciar una escasa confianza en la argamasa y el hormigón, que no llegaron a adquirir la mayoría de las veces la consistencia del romano y no se utilizaron, por tanto, con tanta asiduidad (Ling, 1985, 26; Butler, 1983, 127).

En el acueducto de Nueva Carteya, la cimentación consistía en una tongada de hormigón, (de 1,12-1,25 m. de ancho total), de lado a lado de la estructura, es decir, abarcando todo lo que sería el alzado del specus. En algún punto se ha documentado la existencia de zapatas (sobresalían 13 y $6 \mathrm{~cm}$. en los Pto.3.E y 3.A) que daría una cimentación más ancha que el alzado, pero esto no sucede de forma generalizada. Asimismo hemos comprobado que la cimentación del acueducto apoya sobre la propia tierra o roca del lugar (Pto.3).

A partir de la cimentación propiamente dicha se alzaban sucesivas tongadas de hormigón, de unos $0,50 \mathrm{~m}$. (0,45 a 0,53 m.) (lám. 3b) (11), de forma independiente en los laterales del specus de lo que sería la base del mismo (se documenta de forma clara en el Pto. 2 y se confirma en los Pto. 3D, Pto. 11, Pto. 16 y Pto. 18) (Fig. 3 a y b). Así, entre los dos apoyos de los muros laterales del specus se llevaba a cabo una preparación para el mismo, (documentada en el Pto.2). Consistía en una capa de unos $10 \mathrm{~cm}$. de piedras menudas $(6,5 \times 5 \mathrm{~cm}$.), probablemente para regularizar la base y, sobre ella, una nueva capa de opus caementicium cuidado (de unos $28 \mathrm{~cm}$. de grosor) y que acababa en una cara lisa para asentar el signinum del specus (Fig...3 a).

El specus propiamente dicho, de unos 44 a $50 \mathrm{~cm}$. de ancho según las zonas, estaba flanqueado por dos muretes de $39-40 \mathrm{~cm}$. hasta $48 \mathrm{~cm}$. de grosor. La parte externa se puede ver la terminación de argamasa (documentada en Pto. 3.E y Pto. 15) con huellas de los tablones de encofra-

(11) La disposición del opus caementicium en tongadas fue habitual en Roma y ciudades Itálicas. También se documenta en construcciones de diversas ciudades de la Bética, donde las tongadas suelen medir entre 0,50 y $1 \mathrm{~m}$. de grosor (Roldán Gómez, 1993). 
do (12). No se trata, por tanto, de un revestimiento posterior a la construcción de los muros sino de la propia argamasa del opus caementicium que, quizás de forma intencionada, rebosó sobre la superficie de los caementa.

El interior del specus iba recubierto de signinum (lám.2 b), que se colocaba en primer lugar en el fondo del mismo, de forma independiente a los alzados; sobre él se realizaban los modillones hidráulicos de $8 \mathrm{~cm}$. de altura, y $7 \mathrm{~cm}$. de grosor (Pto.11) (lám. lb) (13). El grosor del signinum es mayor en el suelo, unos $5,5 \mathrm{~cm}$. (Pto. 2) que en las paredes laterales $(1 \mathrm{a} 2 \mathrm{~cm}$., Pto. 2 , Pto. 3D, Pto. 9B y hasta $4 \mathrm{~cm}$. en el Pto. 11, parte inferior). En estas últimas, en ocasiones era aplicado en disminución hacia arriba (Pto. 12 y Pto. 11) de forma que el grosor iba de $3-4 \mathrm{~cm}$. en la base a una fina lechada, que llegaba a unificarse con la argamasa del opus caementicium, en la parte superior.

En algún punto se documenta que la argamasa de los lados interiores del specus fue picada para que el opus signinum pudiera adherirse a ella proporcionando una mayor cohesión (Pto. 11) (lám. lb).

La drástica separación de los laterales y el centro del specus ha hecho que en muchos puntos se haya abierto el acueducto de forma que todo un lateral, el que no apoyaba en la ladera, se ha desmembrado y caído dejando al aire el interior del specus y seccionada su cimentación (Pto. 11) (lám. 1a).

Hemos podido documentar una altura del specus de hasta $47 \mathrm{~cm}$. aunque no podemos estar seguros si ésta sería la altura original ya que no se aprecia el remate de los laterales.

En cuanto a su cubrición, no se conservan restos visibles. Pensamos que podría ir cubierto con tegulae y opus caementicium en sus partes subterráneas y quizás con piedras en sus partes vistas. Unicamente en un punto se documentan dos piedras sobre la estructura hidráulica aunque no están in situ; por su posición, podrían haber caído al romperse la parte superior del acueducto (Pto. 13) (Fig. 3d).

Técnicamente, la conducción debe originarse en fuentes situadas por encima de los $700 \mathrm{~m}$. y mantiene su recorrido por debajo de los $500 \mathrm{~m}$., concretamente entre los 460 y $420 \mathrm{~m}$. en el tramo que hemos documentado. Después, debe bajar aún más al atravesar los llanos de la actual Espejo para remontar de nuevo la colina al llegar a la ciudad. Su recorrido mantiene, prácticamente en todo momento, la misma cota o tiene variaciones mínimas. Ello se consigue siguiendo las curvas de nivel, a las que se adapta mediante sucesivos quiebros y giros. La altura de las fuentes posibilitaba el ascenso del agua hasta la ciudad por el sistema de sifón, habitualmente empleado en los acueductos romanos.

De este modo, el acueducto no tuvo que salvar grandes depresiones, excepto en el tramo final y se evitó tener que realizar obras de envergadura como arquationes, etc. No obstante, debió de cruzar pequeñas vaguadas mediante arcos únicos, probablemente realizados en ladrillo,

(12) La medida habitual de los tablones de encofrado en construcciones romanas es de $20 \mathrm{~cm}$. (Lugli, 1959; Adam, 1989). En la Bética también se documentan medidas similares o algo mayores, entre 20 y $30 \mathrm{~cm}$. y algo más estrechos cuando se trata de bóvedas (Roldán Gómez, 1993).

(13) Este procedimiento de realización del specus, realizando en forma independiente los modillones, se puede documentar en otras construcciones hidráulicas de la Bética. Debió de ser una forma relativamente habitual ya que pueden verse algunos ejemplos similares en ciudades de otras provincias romanas como el acueducto de Augusta Raurica (Grewe, 1988, 67). Otros ejemplos sobre la construcción de specus en varios acueductos pueden verse en esta misma publicación (Die Wasserversorgung Antiker Städte, Mainz, 1988). 
como se ha documentado en uno de los tramos (Pto. 3B) (lám. 2a). En este caso, el arco estaba realizado en ladrillos de dos medidas distintas: $28-30$ x 5-6 cm. y 13,5-19 x $6 \mathrm{~cm}$. (14); arrancan desde el inicio del arco, que parece corresponder a la propia cimentación, y sobre ellos se documenta la masa de opus caementicium que forma el habitual alzado del specus, en el que ha quedado la huella de los ladrillos una vez desaparecidos éstos (Fig. 4). De esta forma aligeraron el muro permitiendo mediante una perforación en arco el paso del agua que debió discurrir con cambios estacionales de caudal.

La base del acueducto se mantuvo en disminución constante de altura por lo que no se realizaron de pozos de nivelación. Solamente hemos documentado una pequeña arqueta de regulación (Pto. 11) (lám. 3a), de planta cuadrada y de pequeño tamaño $(82 \times 82 \mathrm{~cm}$.) que no sobresale sino que queda dentro de la base de cimentación pero que amplía el interior del specus, a ambos lados, casi $30 \mathrm{~cm}$. Sus ángulos son redondeados y está protegido en el fondo y laterales con signinum que forma el característico modillón hidráulico hasta una altura de unos 0,20 m. (Fig. 3c). El canal iría probablemente cubierto con bóveda de opus caementicium o con tegulae (15).

Dada la altura a la que se encuentra la ciudad de Espejo y que el acueducto debió remontar al final de su recorrido, pudo haberse utilizado el sistema de sifón (16). Su realización, en este caso, sería, como se documenta con frecuencia en los acueductos romanos, por medio de tuberías cerradas, consiguiendo de este modo la presión necesaria para posibilitar el ascenso del agua hasta el correspondiente castellum.

Así pues, como hemos visto, el acueducto de Nueva Carteya es una construcción de considerable importancia. Desde la zona de manantiales al sureste de Nueva Carteya, a unos 15 kilómetros de distancia en línea recta, llevaba el agua a la antigua ciudad de Ucubi, hoy Espejo, desarrollando para ello un trazado curvilíneo que implicaba un lógico aprovechamiento de la orografía del terreno.

Poco ha aportado la Arqueología por ahora al conocimiento de esta ciudad que, a juzgar por la obra realizada para su abastecimiento, debió de ser importante. De hecho, sólo conocemos de sus edificios algunas referencias, como son los restos de argamasa, de muros romanos, en el lado oeste de la ciudad y el gran depósito que era conocido con el nombre de "baño de la reina Mora” y "la abujera" (17). Se conocían, además, desde 1935, restos de una lujosa villa hacia

(14) La utilización de ladrillos en esta construcción es un indicio cronológico. La difusión de este material en la Península Itálica tiene lugar a partir de época de Augusto, con algunos ejemplos anteriores (Blake, 1947, 287 ss.). En la Bética se emplearon con seguridad en el s.I d.C., pero no existen evidencias seguras, al menos en las ciudades, de una utilización anterior (Roldán Gómez, 1993).

(15) El tipo de conducción cerrada es necesario, en este caso, en que el specus discurre a escasa altura, para el mantenimiento del agua en condiciones adecuadas para su consumo. Se documenta un tramo de uno de los canales de captación, que iría subterráneo, cubierto con tegulae (Pto.24) y, en otro punto, la existencia de dos piedras caídas que bien podrían responder a la misma función. Ambos tipos de cubiertas están documentados en acueductos de la Bética romana.

(16) Debió de ser un sistema empleado con cierta frecuencia aunque en la mayor parte de los casos no se han conservado. Así, por ejemplo, se documentan nueve sifones en los acueductos que abastecia a la ciudad romana de Lugdunum (Lyon) (Burdy, 1979) y también los hay en Alatri y Aspendos (Leveau, 1979, 17). En Hispania, Fernández Casado cita, entre otros, los del acueducto de Almuñécar, Alcanadre en la conducción de Calahorra y en el puente Zuazo del acueducto de Cádiz (Fernández Casado, 1985, 501 ss). También en este acueducto creemos haber documentado un sifón en el tramo del Cortijo de los Arquillos.

(17) Documentación recogida por Ramírez de Arellano a principios de siglo (Ramírez de Arellano, 1983, 374). Thouvenot, 1973, 528 menciona además unas casas en las que trabajó Romero de Torres y restos de un anfiteatro semiexcavado en la roca (debe referirse a la misma construcción que Ramirez de Arellano consideró un depósito). 
poniente y algunas esculturas que indican, como señaló S. de los Santos, las posibilidades de los ciudadanos de Ucubi (De los Santos, 1942-43, 83-85). Más recientemente se han publicado algunos materiales procedentes de dos sepulturas, por lo que puede deducirse que la zona, al sur del supuesto anfiteatro, se hallaba en el s.I d.C. avanzado, a las afueras del núcleo urbano (Marcos Pous y Vicent Zaragoza, 1988, 200).

Dada la escasez de datos arqueológicos se hace necesario, por tanto, acudir a las fuentes históricos y epigráficas, que tampoco son muy abundantes, para conocer algunos detalles de la ciudad (18). A través de ellas está confirmada su identificación con la actual Espejo (Thouvenot, 1973, 219, Lacort, 1988, 173).

Su organización en Colonia Claritas Iulia Ucubi, se relaciona generalmente con César, en lo que parece haber total unanimidad, a causa del nombre, sin que se sepa nada más de ella en lo que se refiere a su fundación (González Román, 1991,102; Caballos Rufino, 1978, 273 ss.; Abascal y Urbano, 1989, 61-62 y Lacort Navarro, 1991,173). No es citada en las fuentes itinerarias, a pesar de ser un centro comunicado con importantes vías como la de Corduba a lliberri (vereda de Granada) y la que comunicaba Ategua e Spalis (Monturque?) que pudo constituir la vía vecinal de Ucubi (Melchor Gil, 1991,152).

La falta de referencias arqueológicas para el desarrollo de la ciudad hace difícil el establecimiento de una cronología para el acueducto. Parece posible, como han señalado Lacort y Jiménez $(1988,15$; Lacort Navarro, 1991, 176) que el acueducto se construyera precisamente en relación con la organización en colonia de la ciudad y el desarrollo urbanístico que éste hecho llevaría consigo, a finales del s.I a.C. o comienzos del s.I d.C.

La utilización del opus caementicium sin ningún tipo de revestimiento es indicio de cierta antigüedad en Roma, Lugli $(1957,432)$ lo considera anterior a época de Claudio (IV período de la opera caementicia) cuando se generaliza el uso de paramentos externos. Se utilizó preferentemente para cimentaciones, núcleos y bóvedas y, como alzado, se empleó sobre todo en construcciones hidráulicas, ya que, resultaba más adecuada su superficie rugosa para adherir el opus signinum (Lugli, 1957, 415). Así, se empleó en el acueducto augusteo de Cornalvo, en Mérida (Jiménez Martín, 1976, 114) y en otras construcciones de la Bética como los silos del Carchena, de comienzos del s.I d.C. (Lacort Navarro, 1982) el teatro y las termas de Carteia, del s.I d.C. y en la estructura sobre el teatro de Itálica, fechada a comienzos del s.II d.C.

Es posible, por tanto, que haya que considerar una utilización en la Bética del opus caementicium visto, cuando en Roma apenas se daba, como ocurre, por ejemplo en el empleo del opus quadratum, que se utiliza en Itálica en el s.II d.C., cuando ya en Roma no era de uso habitual. Este hecho, junto con la presencia de ladrillos, aunque mínimamente documentados, nos inclina a considerarlo de cronología algo posterior a la propuesta por Lacort Navarro, es decir, a comienzos de la segunda mitad del s.I d.C., cuando la utilización del material latericio se normalizó en la Bética.

La construcción del acueducto de Nueva Carteya implica el aprovechamiento intenso de las tierras cordobesas, como respuesta a las necesidades económicas creadas por una población posi-

(18) Las fuentes epigráficas están recogidas en el CIL II, 1404, 1533, 656 y 1572; en Santos Gener, 1943, 83-85 y Caballos Rufino, 1978, 274. En esta última y en otras publicaciones se recogen además de los datos epigráficos las referencias del Bellum Hispaniense y otras fuentes históricas alusivas a la ciudad de Ucubi (Caballos Rufino, 1978, 274 ss.: Marcos Pous y Vicent Zaragoza, 1988, 193). 
blemente abundante. Ello hizo rentable la realización de una gran obra para aprovisionarse de agua que iría destinada a las necesidades de la ciudad y quizás también a las necesidades agrícolas (19).

Resulta obvio aludir, de nuevo a las posibilidades agrícolas de la campiña cordobesa y a la utilización en época romana de esta zona. De ello tenemos pruebas tan evidentes como las cercanas construcciones agrícolas del Carchena, ya citadas, que documentan la necesidad de almacenar una gran cantidad de grano. Para ello, fue necesario crear toda una infraestructura, desde un cryptopórtico, adecuado para determinadas reuniones, hasta los numerosos silos construidos en una pequeña extensión de terreno (20). En este contexto, el acueducto de Ucubi se encuadra en el desarrollo de la Campiña Cordobesa, acentuado a comienzos del Imperio, cuyo hábitat mantiene una continuidad con respecto a los anteriores núcleos ibéricos, con las necesarias transformaciones urbanísticas (Carrillo, 1991, 104).

Así pues, el estudio de este acueducto aporta nuevos elementos para el conocimiento de la ciudad romana de Ucubi que, no obstante, deberán ser mejor valorados en el futuro con el apoyo de otros datos arqueológicos. Pero además, la existencia de algunos tramos de su recorrido en buen estado de conservación hace posible el estudio de su técnica constructiva. De este modo, contribuye también a ampliar nuestro conocimiento sobre la arquitectura hispanorromana en una de sus facetas básicas: el modo de realización y los materiales constructivos. En este sentido, nos permite la comparación con otros acueductos de ciudades mejor conocidas o que jugaron un papel mas relevante en el desarrollo histórico de la Baética romana, como son los de Cádiz, Itálica, Baelo, etc. (2l).

Por último, el acueducto es una prueba más de la potencialidad arqueológica de la zona cordobesa y asimismo del desconocimiento que aún tenemos de algunas de las ciudades que constituyeron el entramado político-administrativo de la Baética en época romana (22).

(19) En este sentido, véase la opinión de J. Lacort y A. Jiménez, sobre la actividad agrícola de los entornos de Ucubi (Lacort y Jiménez, 1988, 15) que puede ser corroborada arqueológicamente por la existencia de depósitos en las inmediaciones del trazado del acueducto.

(20) Estas construcciones han sido estudiadas por Lacort Navarro, 1982, 171-188.

(21.) El objetivo principal de este y otros trabajos monográficos que venimos realizando es, precisamente, el estudio comparativo de las técnicas constructivas en las ciudades y su relación con las características geográficas, económicas e históricas en general.

(22) En este sentido hay que resaltar la labor de prospección y documentación arqueológica que se viene efectuando en estos últimos años en la campiña cordobesa. De ello son prueba las publicaciones recientemente aparecidas de Encuentros de Historia local en que se recogen los más recientes datos arqueológicos. 


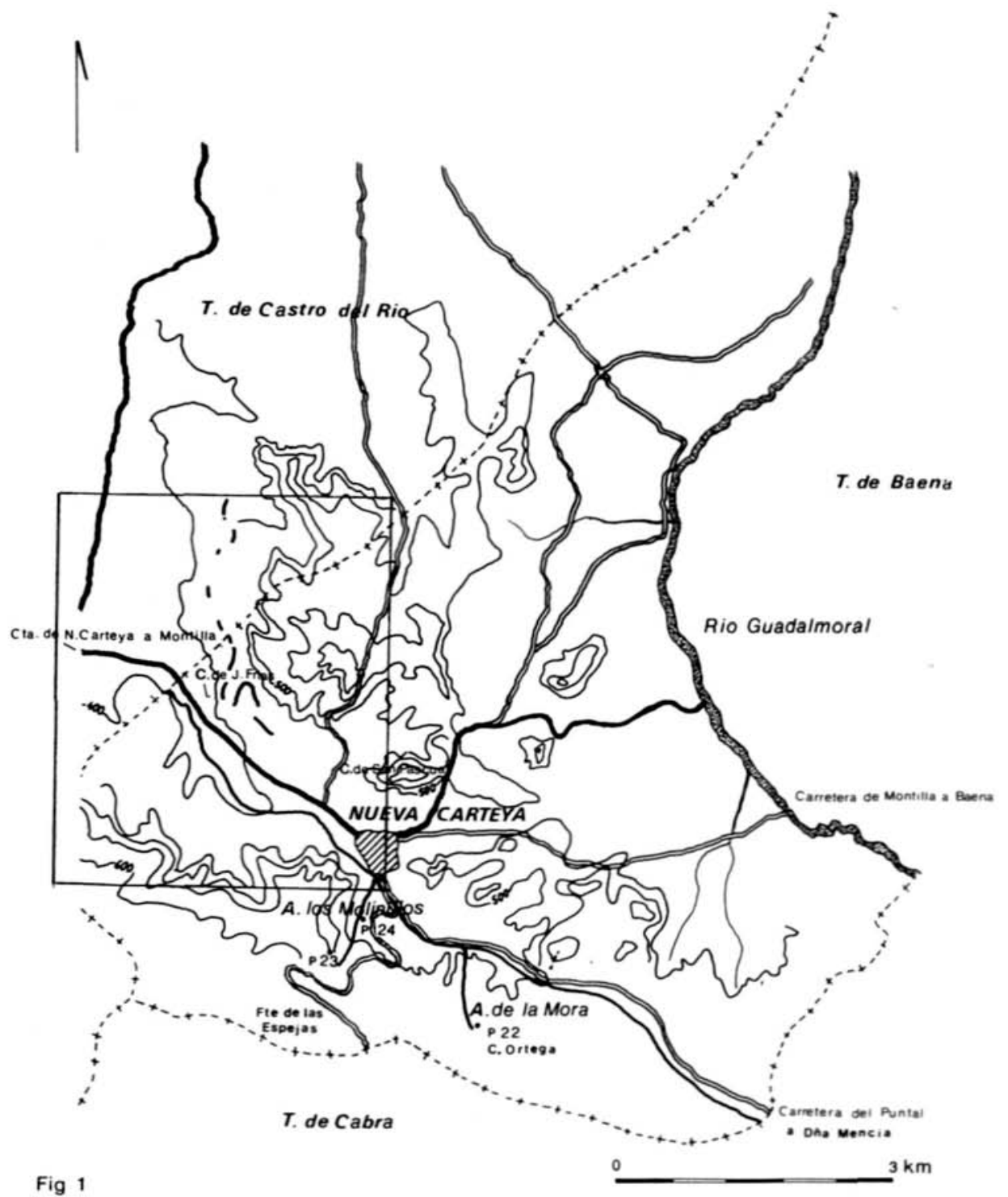

Figura 1: Acueducto de Nueva Carteya. Plano general de ubicación de restos arqueológicos. 


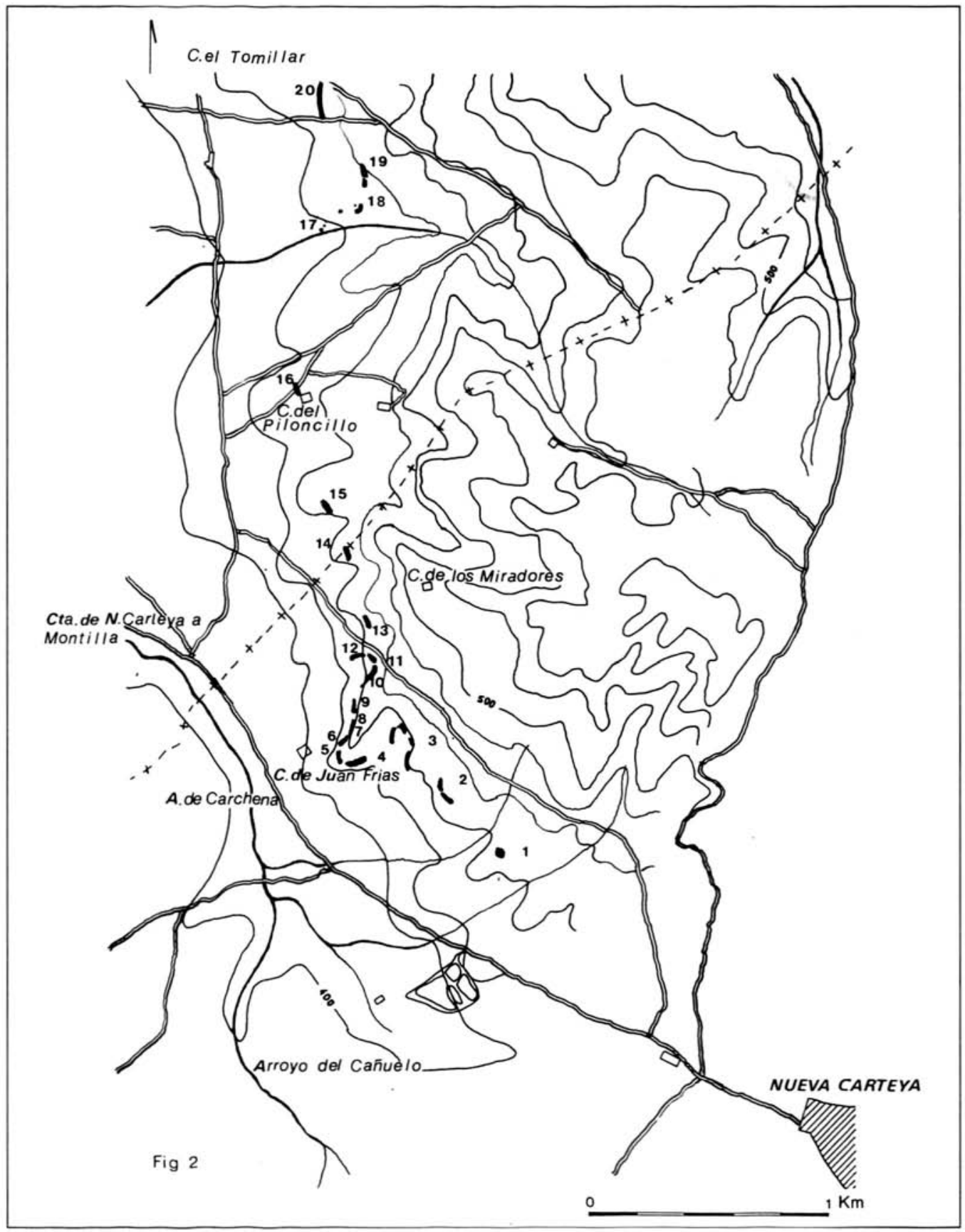

Figura 2: Acueducto de Nueva Carteya. Detalle del recorrido. 

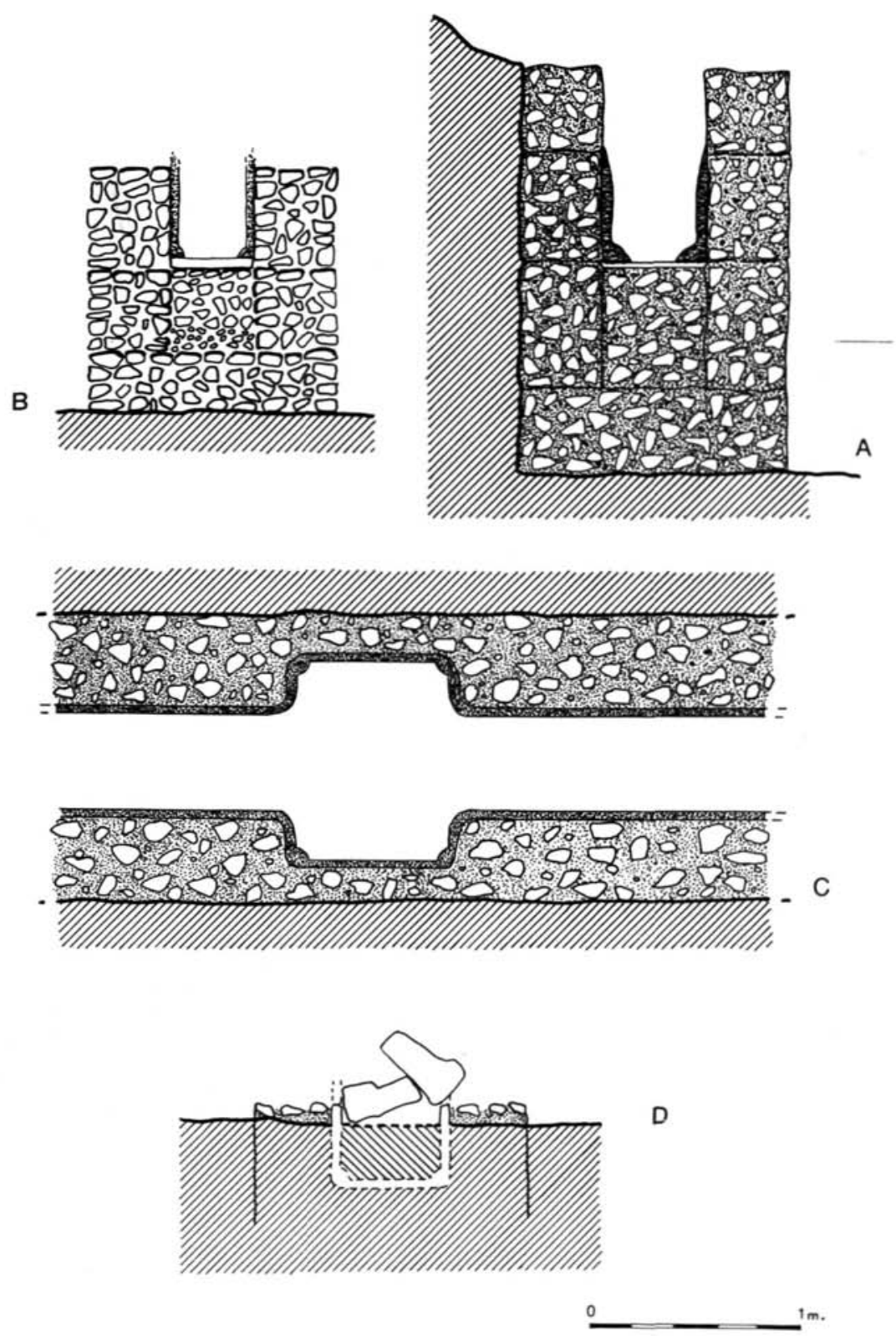

Figura 3: Acueducto de Nueva Carteya. Canal principal. A y B: secciones del specus en diferentes tramos de su recorrido (Pto. 2 y Pto. 11); C: planta de la pequeña arqueta; D: sección del specus y posibles piedras de cubrición (Pto. 13). 


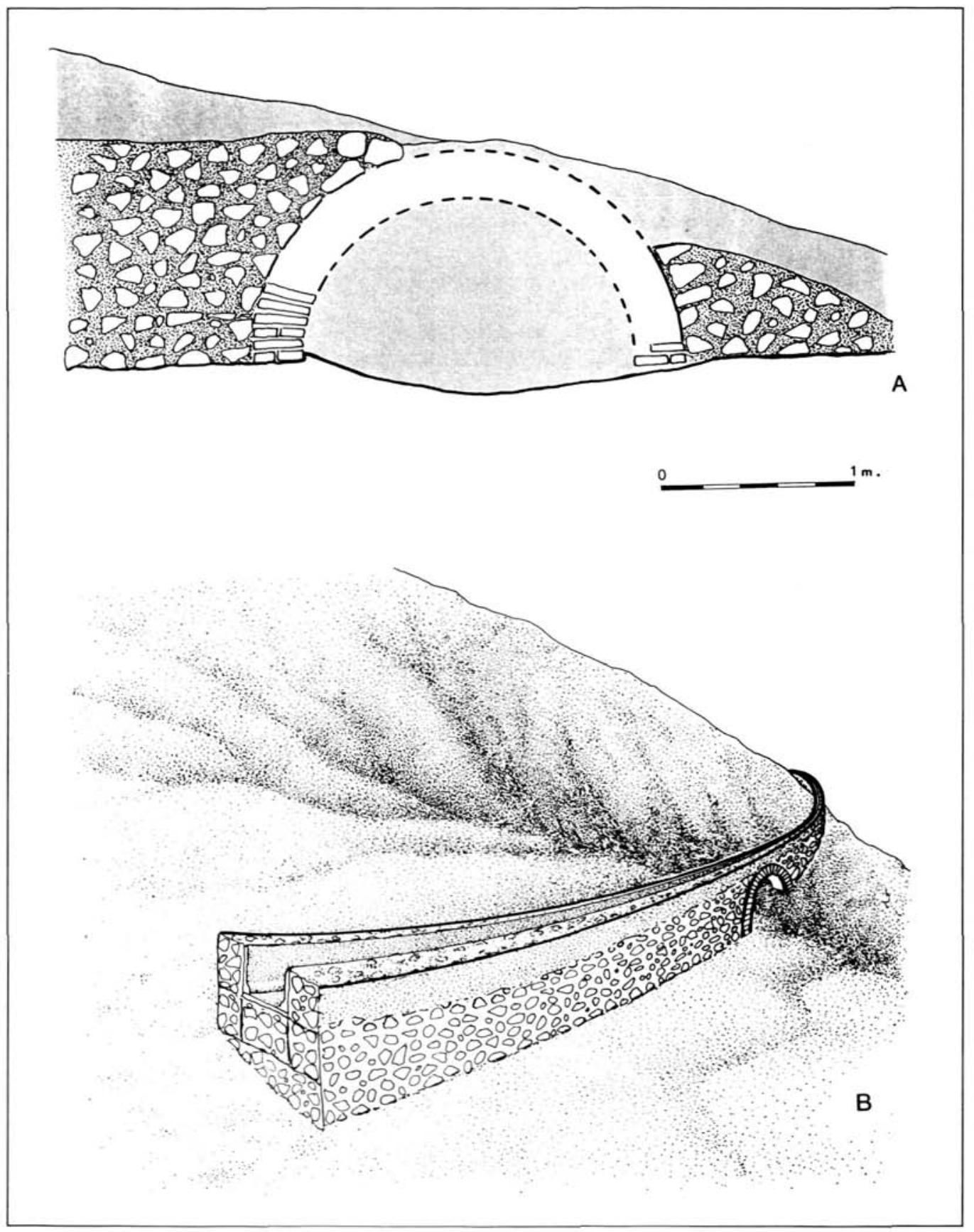

Figura 4: Acueducto de Nueva Carteya. Canal principal; alzado y reconstrucción de la zona del arco (Pto. 3b). 


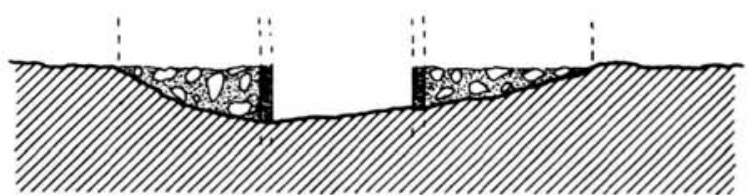

A
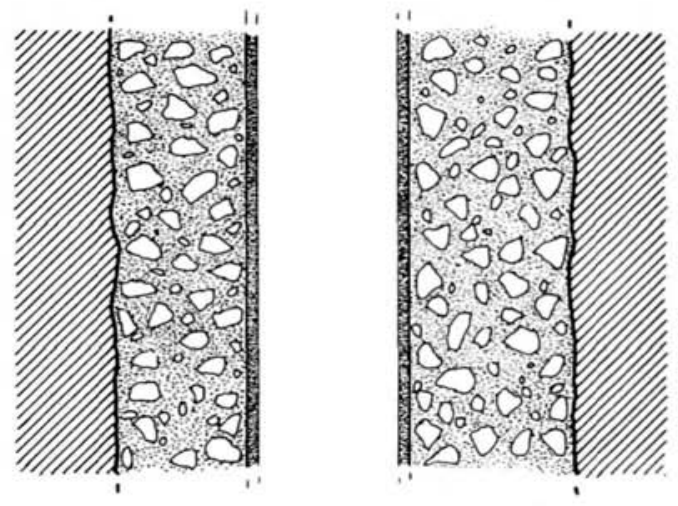

B
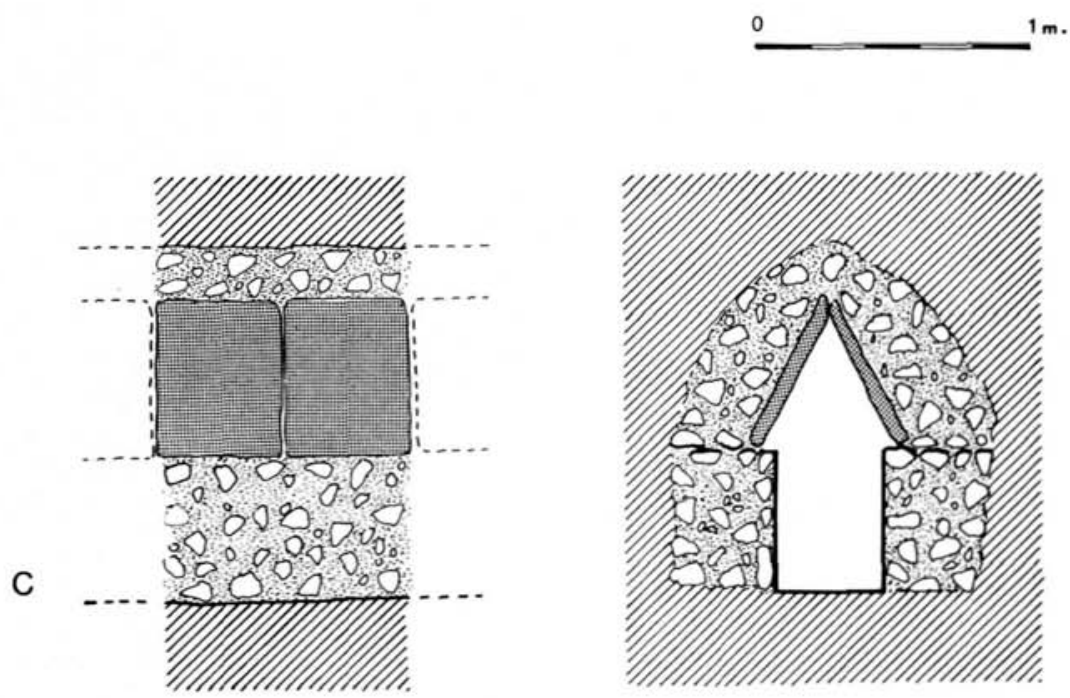

D

Figura 5: Acueducto de Nueva Carteya. A y B: sección y planta del specus del Ramal I (Pto. 22); C-D: sección longitudinal y trasversal del specus del Ramal II (Pto. 24), sector con cubrición de tegulae 


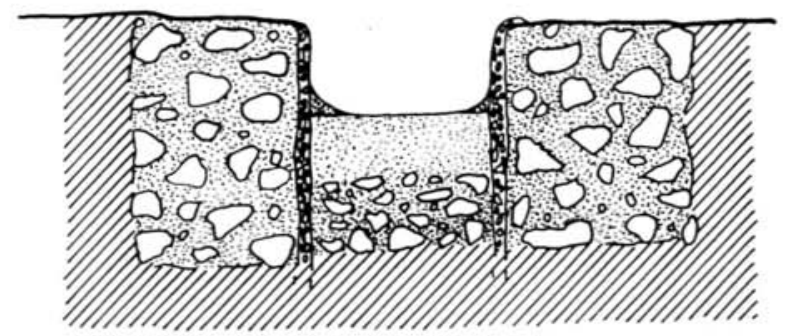

A
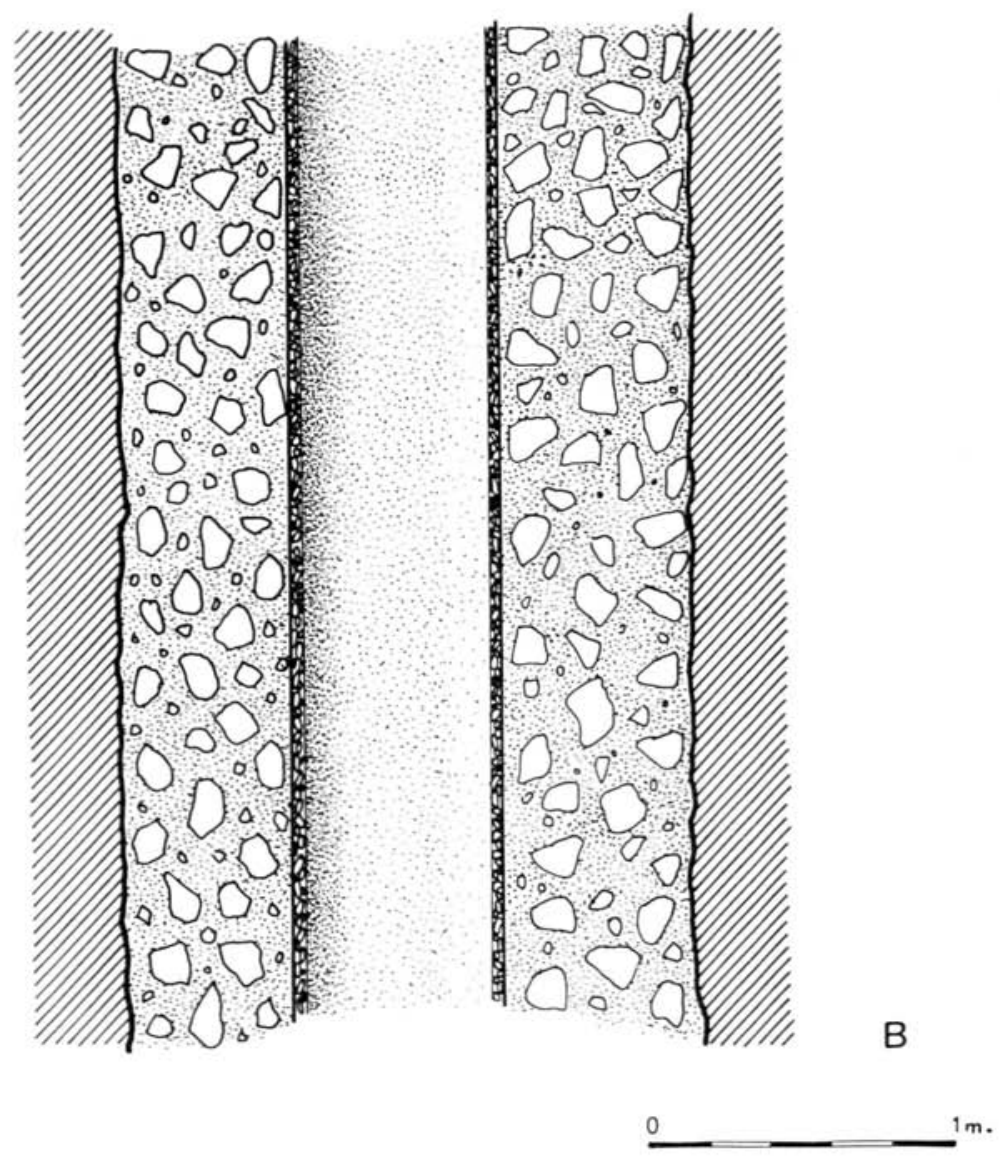

Figura 6: Acueducto de Nueva Carteya. Planta y sección del specus del Ramal II (Pto. 23). 

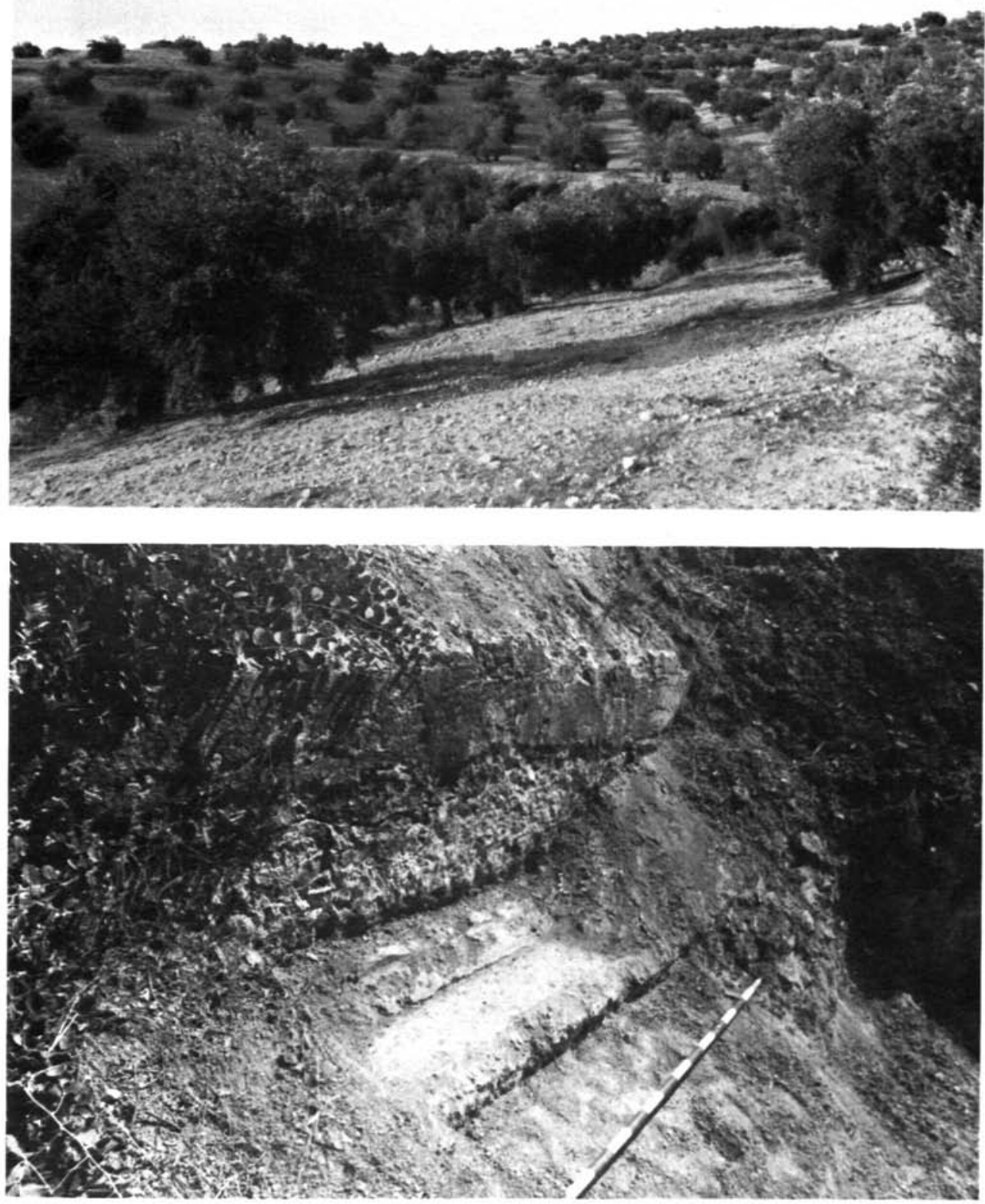

Lám. 1. a: Zona de olivares por donde discurre el acueducto. b: Interior del specus (Pto. 2). 

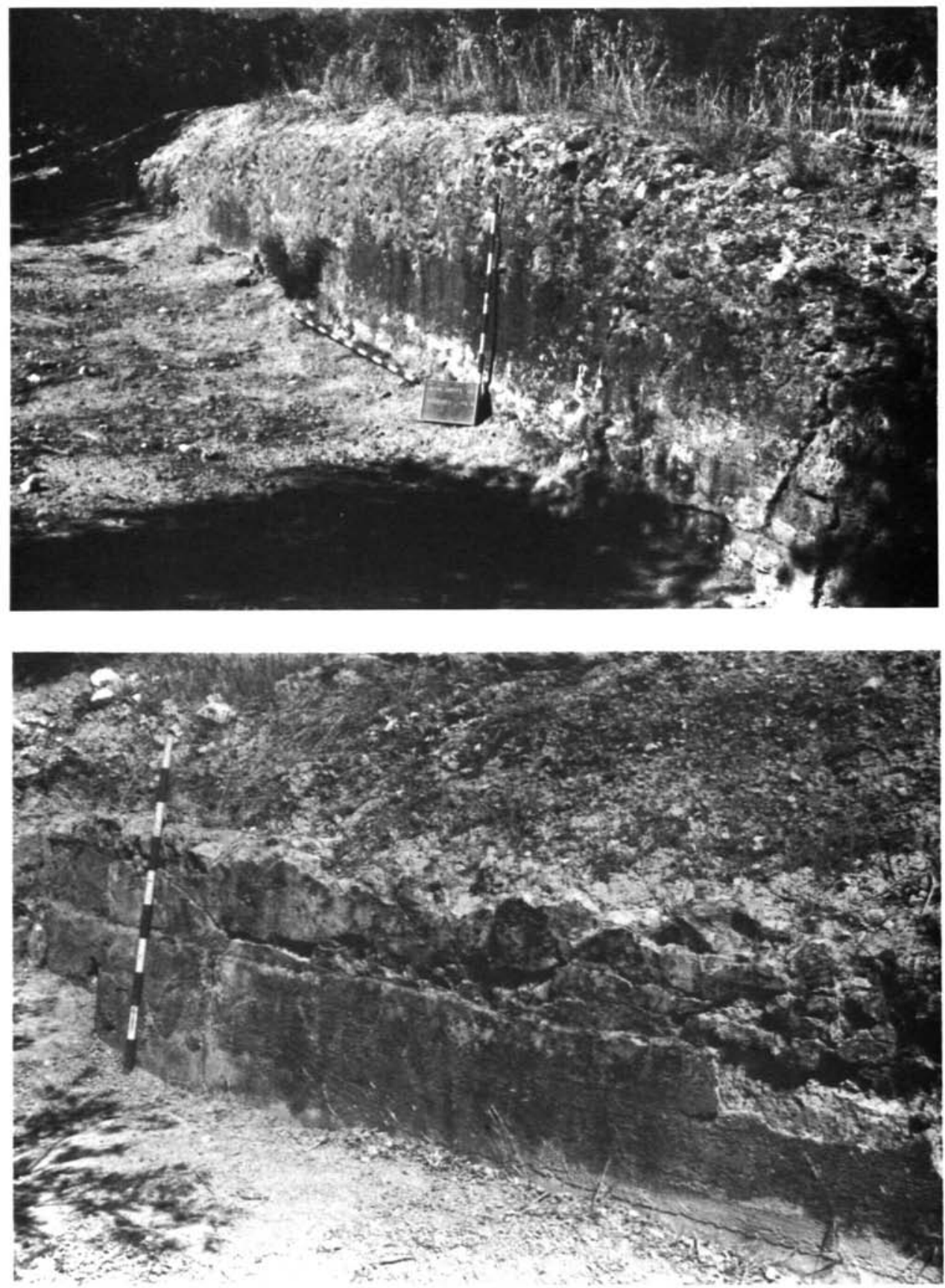

Lám. 2. a: Alzado del muro externo del specus y arco de ladrillos. b: Interior del specus recubierto de opus signinum (Pto. 11). 

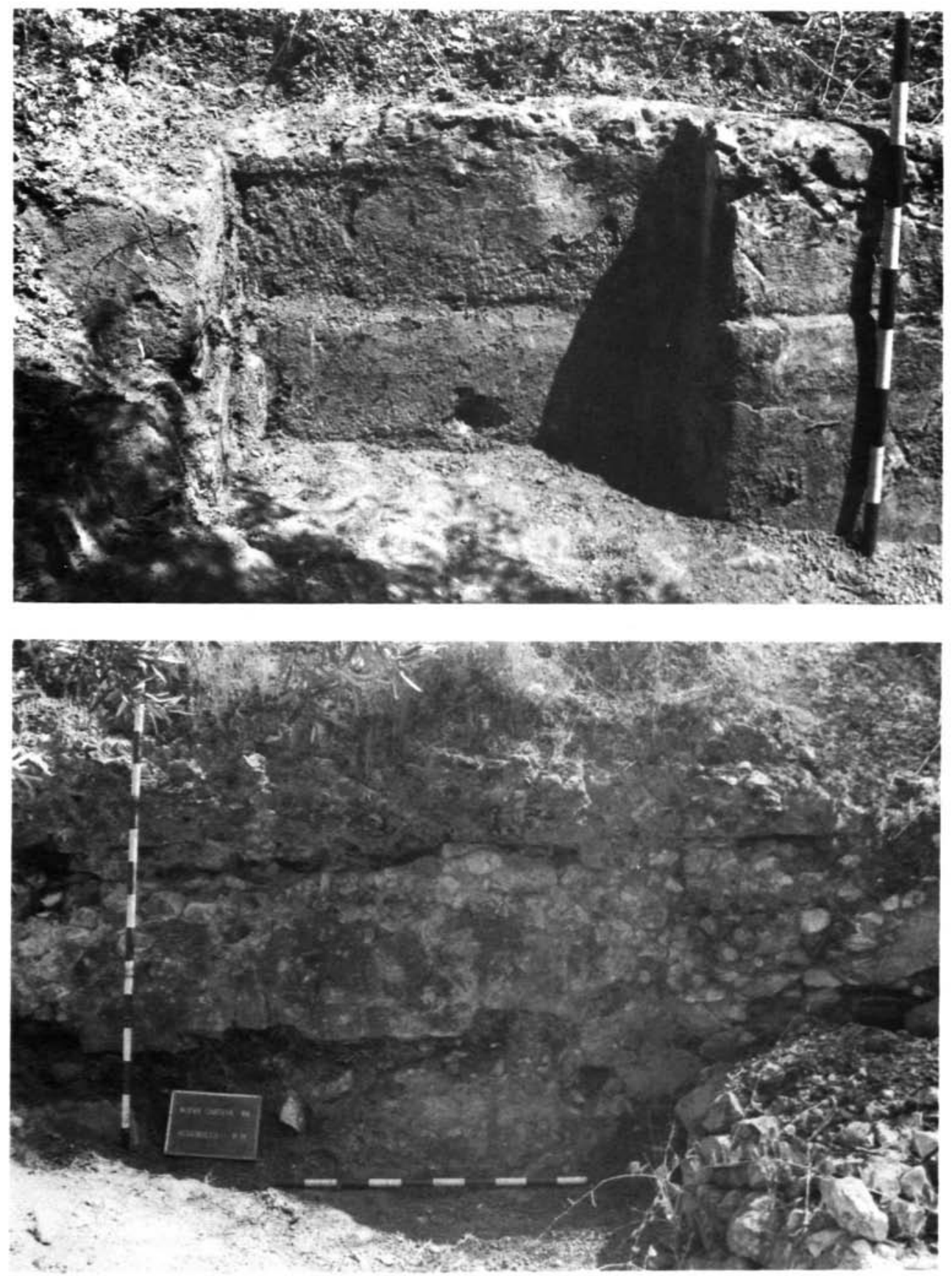

Lám. 3. a: Pileta de decantación con modillón hidráulico en las esquinas (Pto. 11).

b: Tongadas de hormigón del alzado externo (Pto. 11). 


\section{BIBLIOGRAFIA}

ADAM, J. P., 1989: La construction romaine. Materiaux et techniques, Paris.

ABASCAL, J. M. y U. ESPINOSA, 1989: La ciudad hispano-romana, privilegio y poder, Logroño.

BlaKE, M. E., 1947: Ancient Roman constructions in Italy from prebistoric period to August, Washington.

BURDY, J., 1979: "Les quatre aqueducs de Lyon capitale des Gaules", Archeologie. 38, 62-73.

BUTLER, R. M., 1983: "The construction of Urban Defences", Roman Urban Defences in the West, London, pp. 125-129.

Caballos Rufino, A., 1978: "Colonia Claritas Iulia Ucubi", Habis. 9, pp. 273-291.

Catálogo, 1985: Catálogo Artístico y Monumental de la provincia de Córdoba T. II, Córdoba 1985.

COARelli, F., 1987b: I Santuari del Lazio in etá Republicana. Roma.

De los Santos, S.: "Monumentos romanos de Claritas Iulia Ucubi (hoy Espejo), en el Museo Arqueológico de Córdoba", Memorias de los Museos provinciales III-IV, 1942-1943, pp. 83-85.

Die Wasserversorgung, 1988: Die Wasserversorgung antiker stadte, Mainz.

FERNANDEZ CASADO, C.: Ingenieria bidráulica romana. Madrid, 1985.

GiUlıani, C. F., 1990: L'Edilizia nell antichitá. Roma.

González Roman, 1991: La Bética en su problemática bistórica. Granada.

GREWE, K., 1988: "Römische Wasserleitungen nördlich der Alpen", Die Wasserversorgung Antiker Städte. Mainz, pp. 45-97.

GullıNI, G., 1989: "Tradizioni e innovazione nelle fasi edilizie del Santuario della Fortuna Primigenia tra el III e il Is. a.C.", Urbanistica ed Architettura dell'antica Praeneste, Palestrina, pp. 69-86.

Jiménez Martín, A., 1976: "Los acueductos de Emérita", Augusta Emérita. Actas del Bimilenario de Mérida. Madrid, pp. 41-125.

Lacort Navarro, P., 1982: "Sobre las construcciones romanas del Carchena (T.M. de Castro del Río, Córdoba)", Habis 13, pp. 171-188.

... 1989: "Obras hidráulicas e implantación rural romana en la campiña de Córdoba", El agua en zonas áridas: arqueologia e historia. I Coloquio de Historia del Medio Físico, (Almería, 1989) Almería, pp. 361-401.

... 1991: "El contexto arqueológico romano en el territorio de Ucubi (Espejo)", II Encuentros de Historia Local. La Campiña. Córboba, pp. 173-190.

LACORT NAVARro, P. y A. JimÉnez RUIZ, 1988: “Un acueducto romano en los términos municipales de Nueva Carteya, Castro del Río y Espejo", Caja Sur. 1988.

LeVeau, Ph., 1979: "Les techniques de construcción des aqueducs, Archeologie. 36, pp. 8-19.

LUGLI, G., 1959: Tecnica edilicia romana. con particolare riguardo a Roma e Lazio, Roma.

LING, R., 1985: "The mechanics of the Building Trade" Roman Urban Topography in Britain and the Western Empire, Londres, pp. 14-27.

Marcos Pous, A . y A. M. Vicent Zaragoza, 1989: "Sepulturas romanas de Ucubi: actual espejo (Córdoba)", Homenaje a D. S. de los Santos, Albacete, pp. 193-204.

MelChOR GIL, E., 1991: "La red viaria romana de la campiña de Córdoba. Sector oriental", II Encuentros de Historia local. La Campiña. Córdoba, pp. 151-172.

Morena, J. A., M. SÁnChez de la ORden y A. García Ferrer, 1990: Prospecciones en la Campiña de Córdoba, Córdoba.

Ramírez DE AREllano, 1983: Inventario Monumental y Artístico de la provincia de Córdoba, Córdoba (con notas de J. Valverde).

Ramírez de las Casas Deza, L. M., 1986: Corografía Histórico-Estadística de la Provincia y Obispado de Córdoba (estudio introductoria y edición por A. López Ontiveros), Córdoba.

Roldán Gómez, L., 1992: Técnicas Arquitectónicas en la Bética Romana. Tesis Doctoral. inédita.

ThOUVEnor, R., 1973: Essai sur la province romaine de Bétique, París. 